\title{
E-INFRASTRUCTURE SUPPORT FOR NANOCMOS DEVICE AND CIRCUIT SIMULATIONS
}

\author{
R.O. Sinnott, G. Stewart \\ National e-Science Centre, University of Glasgow \\ A. Asenov, C. Millar, D. Reid, G. Roy, S. Roy \\ Dept of Electronics and Electrical Engineering, University of Glasgow \\ C. Davenhall \\ National e-Science Centre, University of Edinburgh \\ B. Harbulot, M. Jones \\ Research Computing Services, University of Manchester \\ r.sinnott@nesc.gla.ac.uk
}

\begin{abstract}
The UK e-Science EPSRC pilot project Meeting the Design Challenges of nanoCMOS Electronics (nanoCMOS - www.nanocmos.ac.uk) was funded to address the challenges facing the global electronics semiconductor industry caused by the decreasing size of Complementary Metal Oxide Semiconductor (CMOS) transistors and the atomic variability present in devices manifest at these dimensions. Fundamental problems to be addressed include the modelling, understanding and predicting the effect of differences in the atomic structure of devices on their behaviour, and then using this information to guide electronic circuit and system designers who utilise CMOS components. In this paper we describe the e-Infrastructure that has been developed as part of the nanoCMOS project and outline how it supports large scale high performance computing (HPC) simulations of ensembles of devices which can subsequently be used to model and understand the impact that they have on very large electronic circuits. Key features of this e-Infrastructure include support for very large scale HPC utilization; dealing with federated data sets and associated metadata from multi-level simulations, and addressing challenges related to security and intellectual property protection of data, simulation codes and electronic designs as a whole.
\end{abstract}

\section{KEYWORDS}

nanoCMOS electronics, high performance computing, data management, security.

\section{Introduction}

The UK e-Science EPSRC pilot project Meeting the Design Challenges of nanoCMOS Electronics (nanoCMOS - www.nanocmos.ac.uk) [1] was funded to address the challenges facing the electronics industry caused by the decreasing atomistic dimensions of Complementary Metal Oxide Semiconductor (CMOS) transistors. Historically, all transistors could be regarded as having uniform properties. However, with the decreasing scale of CMOS devices, this assumption is no longer true and microscopic differences in the number and position of individual dopant atoms, caused by the inherent discreteness of charge and matter at these scales, is beginning to affect the macroscopic behaviour of individual transistors. As a result Moore's Law as tracked by the International Technology Roadmap for Semiconductors (ITRS - www.itrs.net), and semiconductor designs are now approaching fundamental physical, atomic limits of silicon and new approaches are needed which are capable of coping with this unavoidable variability in device behaviour throughout the electronics design process. This is non-trivial task given that the design process is itself extremely complex with modern chips now comprising several billions of individual transistors.

This problem is not an insurmountable one however. It is quite possible to simulate the expected behaviour of microscopically varying devices to understand and predict for example the upper and lower limits on their differences, e.g. variance in parameters such as their threshold voltages. However, such simulations are computationally expensive to run, since very large ensembles of 3-dimensional atomistic simulations must be performed for each transistor. These can include: drift diffusion simulations which require the solution of coupled partial differential equations (PDEs) consisting of the Poisson, current continuity and density gradient equations discretised on meshes typically comprising millions of nodes; Monte-Carlo simulations which couple the mesh based solution of the Poisson equation to classical particle trajectories, interrupted by stochastic scattering events, and as devices continue to shrink in dimension and quantum mechanical effects begin to have an impact upon electron transport, solutions of the Poisson equation coupled to recursive algorithms for single electron Non-Equilibrium Green's functions (NEGF) are required.

These techniques allow the prediction of the behaviour of transistors in response to the specific atomic structure of the device which typically comprises changes in current/voltage $(\mathrm{I} / \mathrm{V})$ response of the device. These individual simulations are computationally expensive and 
require access to large scale high performance computing (HPC) facilities such as the UK e-Science National Grid Service (NGS - www.ngs.ac.uk), the ScotGrid infrastructure (www.scotgrid.ac.uk) and a variety of other heterogeneous HPC resources. The nanoCMOS Geronimo simulator has been developed to simplify the simulation of transistor devices when run on heterogeneous HPC resources.

In the electronics design process, the I/V characteristics that are obtained from the simulation of large ensembles of devices; parameters describing device behaviour can then be extracted and used to construct much simpler mathematical models of each device (socalled "compact models") which are more suited for the simulation of circuits, providing a accurate approximation of device operation at a significantly lower computational cost. This information can then be used by designers in order to construct large electronic designs which fit within particular power, speed, yield and reliability constraints. To facilitate this process, nanoCMOS has developed the RandomSPICE simulator that allows the Monte-Carlo analyses of the impact of device variability on circuit designs to be performed on HPC resources.

Through the application and integration of Geronimo and RandomSPICE simulations, it is possible to show how variability-aware circuits can be simulated and designed and to outline the problems that can arise. Achieving this requires access to more than just large scale HPC resources. Both the Geronimo and RandomSPICE simulators generate significant amounts of data in multiple streams, which must be captured and annotated in order to facilitate analysis.

Unlike other domains, the electronics industry does not have widely agreed international standards on data and meta-data formats. Instead, many of the de facto standards are largely based around legacy, and proprietary file formats and those that specific commercial tools support. To address these issues, the nanoCMOS e-Infrastructure supports a variety of data services for the capture of user specified simulation meta-data and to provide seamless, secure access to distributed file-based data sets. Often this data has intellectual property associated with it and thus requires secure, discretionary access control.

In this paper we focus upon the associated eInfrastructure supporting the nanoCMOS electronics research process. The rest of this paper is structured as follows: Section 2, outlines the Geronimo and RandomSPICE applications. Section 3 outlines the nanoCMOS computational e-Infrastructure for job creation and submission to heterogeneous HPC resources. Section 4 outlines the nanoCMOS data eInfrastructure for data and metadata management. Section 5 outlines the security systems in place to ensure data and computational security. Section 6 describes a case study of the e-Infrastructure in the simulation of a variability-aware NAND gate. Finally in section 7 we draw some conclusions on the work as a whole, outline the usage and uptake of the nanoCMOS e-Infrastructure by the electronics research community and outline areas of future work for the project as a whole.

\section{Variability-aware Device and Circuit Simulations}

Transistor device variability is primarily caused by the discreteness of charge and matter [2,3]. This variability now accounts for more than $50 \%$ of the total variability in current 45nm devices [4]. As devices shrink further, this variability is expected to be further exacerbated [5]. Device variability already profoundly affects SRAM design [6], and causes statistical timing problems [7] and hard digital faults [8] in logic circuits. Understanding the statistical distribution of variability in ensembles of devices is at the heart of understanding device variability. In particular understanding the upper and lower limits of statistical distributions of variability is essential (the tails).

For electronic devices, bulk MOSFETs continue to be the cornerstone of CMOS, and as such nanoCMOS has focused on understanding how variability affects conventional architecture MOSFETs. Until recently, variability analyses of MOSFETs through simulation tended to focus on relatively small samples of devices, typically around 200 [9,10]. With such small samples involved, it is clear that statistical distributions of device variability will not be as accurate as they might (should) be. Considering the latest chips, which contain more than 2 billion transistors, it is necessary to design reliability / tolerances of transistor to 1 failure in $10^{6}-10^{7}$ transistors. Furthermore, since it cannot be predicted where these devices will occur on any given chip, it is necessary to design systems that are suitably tolerant to these variations. This in turn demands that detailed prior knowledge of how transistor device parameters vary.

To support this atomic variability, the nanoCMOS project and in particular the Device Modelling Group at the University of Glasgow, developed the Geronimo simulator [10]. This simulator supports drift/diffusion (DD) which allows to accurately model transistor characteristics in the sub-threshold regime, making it well suited for the study of threshold voltage $\left(\mathrm{V}_{\mathrm{T}}\right)$ fluctuations. The simulator itself includes Density Gradient quantum corrections [11], which accurately capture quantum confinement effects and are essential for preventing artificial charge trapping in the sharply resolved Coulomb potential of discrete impurities. Each device is fully independent, allowing the problem to be easily parallelized using a task farming approach. Each Geronimo simulation can often run from days-weeks on a typical HPC resource such as ScotGrid. The Geronimo simulator was used in simulation studies in nanoCMOS associated with the $35 \mathrm{~nm}$ gate length transistors based on data published by Toshiba [12] with structural data for the device from commercial TCAD process simulation. More details on the Geronimo simulator and its application to characterise the variability of Toshiba 35nm devices is described in [13].

As a result of the statistical variability at the transistor device level, it is necessary to apply a similar statistical approach at the circuit level in order to determine the effects of device variability on important circuit parameters such as delay and power. Characterisation of variability at the circuit level requires accurate compact 
models to be extracted from device simulations, which may then be used in circuit/system simulators such as the widely used SPICE simulator [14]. One of the primary concerns for these simulations is ensuring the correct statistics are transferred from the device level. The compact models extracted from device simulations are for minimal width, i.e. square geometry devices. Unfortunately, simply using larger width values for the transistors the SPICE circuit is not sufficient to capture the correct statistics. Wider devices exhibit lower variability due to self-averaging, which is not handled by SPICE.

To address this, the RandomSPICE application was developed to automate the process of selecting random compact models for the transistors in a SPICE netlist (which represents the overall connectivity of the components (transistors/blocks etc) as used in an electronics circuit design. In using RandomSPICE, devices which have a width to length ratio $>1$ are broken down into sub-circuits containing int(width/length) transistors in parallel.

In order to characterise a circuit in the presence of transistor variability, it is necessary to simulate a statistical ensemble of the circuit in question. To begin with, an ensemble of compact models is extracted from device level simulations generated by Geronimo. Characterisation of a circuit is then achieved by randomly selecting a compact model from the ensemble for each transistor in the circuit and simulating the circuit in SPICE. From here, information such as timing and power consumption may be calculated.

While SPICE simulations themselves are relatively fast (in comparison to Geronimo simulations) each circuit must draw a new set of compact models from the ensemble. Since this is a laborious task to perform by hand, especially for non-trivial circuits, RandomSPICE was developed to automate this process.

To prepare a template netlist for RandomSPICE, specific tags (ATOMN and ATOMP) are used to indicate which transistors should be randomized. These are parsed and replaced by RandomSPICE as shown in Figure 1, producing $n$ random netlists from a single template input (where randomly selected transistors 125, 20, 54, 90 and 5 and 143 have been selected from the ensemble). Figure 2 also illustrates the expansion of transistors into multiple parallel devices. In order to ensure that any given circuit can be reproduced, the random number generator used internally can be seeded from the command line in RandomSPICE.

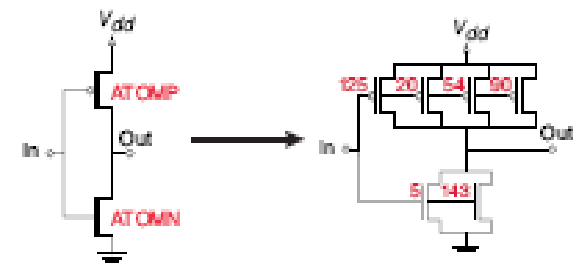

Figure 1: Tagging and Random population of Circuits with Transistors from Device Ensembles

To allow test beds to be implemented without affecting the measurements of variability, further tagging is used (UNIFN and UNIFP). This indicates transistors that should be substituted with the uniform models for the technology being used. An example netlist showing definitions for two inverters built from variable and uniform devices is shown in Figure 2.

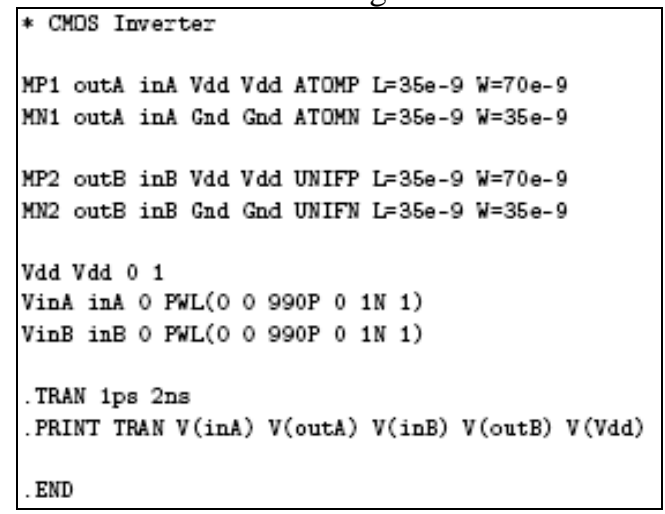

Figure 2: Randomised CMOS Inverter

This shows the voltage drain for two inverters, one of which is based upon a randomly selected compact model from an ensemble of models. In this circuit, the voltage drain varies from 0 to 1 , and the simulation time steps go up to 2ns with 1ps time increments. The actual simulation itself is based upon running this parameterised netlist through ngSPICE (an open source version of SPICE) that runs on HPC facilities such as the NGS.

\section{3. nanoCMOS Computational e- Infrastructure}

The infrastructure developed to support job creation and submission within the nanoCMOS project comprises a number of web services, each of which provides a particular category of functionality (e.g. creation of a particular type of job, or submission to a particular type of resource). These take the form of Apache Axis2 (http://ws.apache.org/axis2) SOAP-based services, with Apache Rampart (http://ws.apache.org/rampart) providing message-level security (encryption, message signing and time-stamping) on all communication between client and service. A series of command-line client applications, distributed in one self-contained bundle, have been written to provide an easy way to interact with these services. The typical flow of interactions in creating a job through the nanoCMOS e-Infrastructure is depicted in Figure 3.

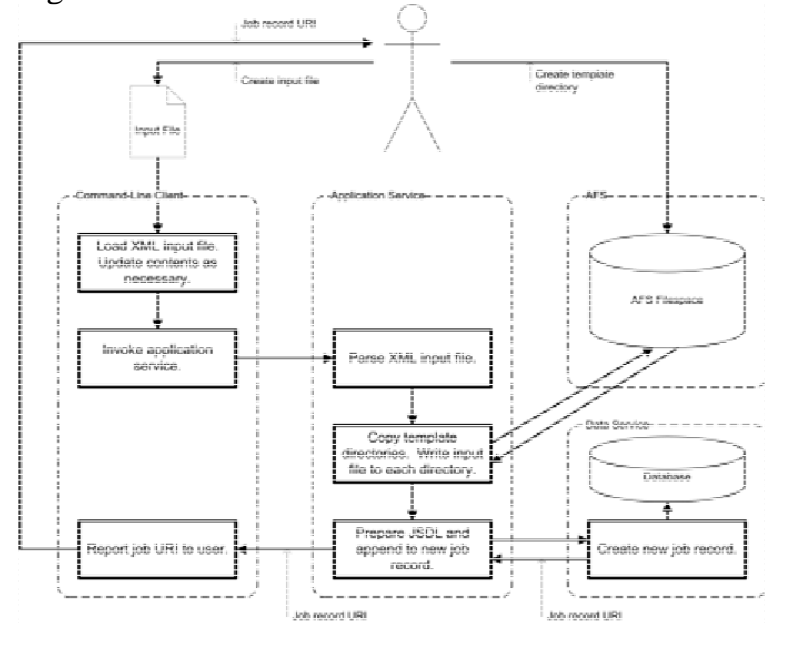


Figure 3: nanoCMOS Job Creation Control Flow

A user is presented with targeted XML-based templates that allow categorisation of aspects of the job itself. This can be based around the number of jobs and associated sub-jobs that are to be created, or to influence the atomistic parameters associated with the Geronimo simulator for example. As shown in Figure 4, the job creation is integrally linked with the data management system which itself exploits the Andrew File System (AFS) [15] as described in section 4.

Running a job through the infrastructure involves two steps: an application service is invoked which has responsibility for creating the job; following this, the job is submitted to a particular HPC resource, either by way of a submission service, or by direct Globus submission.

The e-Infrastructure currently provides three application services, supporting Geronimo and RandomSPICE, as well as arbitrary simulations defined by the user. Although there are inevitable internal differences due to the fact they support different applications, the external interface is common to all application services, and so the same sequence of commands can be used to create and run jobs of any type.

As shown in Figure 3, in order to create a job, a user passes an XML input file to the application service; this file is a modification of the input file passed to the underlying application, and contains additional information required to prepare the job for submission to an HPC resource, such as the desired number of subjobs, i.e. the number of constituent units of work, each of which could, depending on the job manager on the target resource, be executed in parallel, the number of simulations per sub-job, and the version of the application to run. Additionally, this file contains a reference to a template directory on AFS; this directory is created by the user, and contains any files required by the simulation.

On receipt of a request to create a job, the application service duplicates the template directory once per subjob, writing a customised input file to each; this input file is based on that provided by the user, but modified in order that each sub-job will perform its designated portion of the complete simulation. Finally, a new record is created in the data service, containing a description of the job written in the Job Submission Description Language (JSDL) [16], with information about sub-jobs encoded using the JSDL Parameter Sweep Extension [17]. The JSDL is structured such that it can be used with the AFS wrappers described above. The URI of this record is returned to the user. This becomes the identifier by which the job will forevermore be referenced.

Following the successful creation of a job, a user can invoke the submission client application, passing it the job's URI. The client application presents a list of supported execution resources to the user, from which the user selects where the job will run. Once a job has been submitted, additional information necessary to interact with the job in the future-including the resource to which it was submitted, and any resource- specific identifiers that may be required-is written to the job record. At present, submission to clusters running Sun Grid Engine is supported by means of a bespoke submission service we have developed, while the JGlobus module of the Java CoG kit (http://dev.globus.org/wiki/CoG_jglobus) is used to support submission to Globus resources. Regardless of the chosen resource, all further interaction through the client, e.g. status queries or cancelling jobs, is performed in the same way.

Much time has been spent working with end-users of the client applications to ensure that the software developed meets their needs effectively. All the clients are provided in a single archive which includes an installation script to perform all necessary configurations. In order to further simplify matters, any requisite security steps, e.g. generation of appropriate Globus or VOMS proxy credentials, are incorporated within the client. It is not necessary for users to install Globus or any other similar package prior to using the clients.

Using the job URI created in the job creation phase and a targeted command line client, users are offered a collection of resources where the jobs and associated sub-jobs can be executed. Currently we have targeted primarily Globus-based resources such as the nodes of the UK e-Science NGS and Sun Grid Engine-based clusters hosted at the nanoCMOS electronics partner sites. Work is on-going to integrate gLite-based resources into the resources that are available. If the resource is a Globus-based resource, then the job record and associated JSDL is returned, and the associated Globus Resource Specification Language (RSL) syntax is generated for job submission. Job submission itself appears largely the same as a typical Globus-based job submission from the resource provider perspective, with the proviso that the credentials that are used incorporate Virtual Organisation Membership Service (VOMS) attributes used by the resource providers through use of Local Centre Authorization Service (LCAS) and Local Credential Mapping Service (LCMAPS) [18] which map the nanoCMOS user role information into group identities (gid) and user identities (uid) on associated local pool accounts established on the local cluster for that particular nanoCMOS VO. Furthermore, information (tokens) required by the resource providers to access and write to the appropriate AFS directories (see section 5) are used by AFS clients installed on the resource providers.

In the case of non-Globus based resources such as Sun Grid Engine-based clusters, the submission system uses information in the JSDL file that allows direct qsubbased submission to the SGE cluster headnode.

It is worth noting that the clients themselves are primarily command-line based. The original prototypes in the project exploited portal-based clients as outlined in [19]. However, based on feedback from the researchers themselves, it was suggested that command-line based solutions were preferred.

In terms of the functionality offered by the Geronimo and RandomSPICE job creation and submission systems numerous observations are worth noting. Firstly, the solutions developed required bulk job submission. Due 
to the nature of the research and simulations required, it was necessary to execute hundreds-thousands of separate simulations and track these independently. In this process, it was recognised that particular simulations might fail, e.g. the algorithms do not converge. As such it was required to uniquely identify jobs and associated sub-jobs, e.g. for subsequent resubmission for example. In Globus-based systems using JSDL, this bulk job submission was not directly supported other than for example through hundreds or thousands of separate independent globus-job-submit, which has considerable overheads. The issue was one of identification of the jobs themselves when they eventually arrive in a cluster queue. This was a moot point with SGE based clusters as job identification was supported directly.

In using RandomSPICE in a cluster environment there were various significant technical concerns that required addressing. For simulations of small circuits, the primary bottleneck in performance and stability of simulations was disc access. In general, most file systems handle large numbers of small writes poorly, resulting in the writing of output data becoming the bottleneck, rather than the simulations being bound by the computational power available. To alleviate this, RandomSPICE was refined to support a burst write-mode, adding the option of storing results in a single .tar file rather than $n$ individual files. Files are also cached to temporary directories while RandomSPICE is running to reduce network traffic and the strain on networked file systems.

This implementation requires the storage of all runtime data in memory while RandomSPICE is executing. For a moderately large number of small circuits in a single ensemble $(<10,000)$, this is of little concern, however this becomes a major issue for larger circuits, resulting in the requirement of 10's-100's of GBs of memory to store the data if the ensemble size has been selected without due care. The storage requirement becomes increasingly large due to the splitting of transistors into multiple devices and the subsequent storage of the corresponding compact models in the netlist. The implementation of 'width-n' models has reduced the overall transistor count once splitting has been done, thus fewer models need to be stored. As the disc writes associated with larger circuits are larger and occur more slowly, the data associated with all circuits in the ensemble need not be held in memory until the end of execution. To this end, RandomSPICE implements 'batch' simulation mode for small circuits where data for all circuits is held in RAM until completion; and 'interleaved' mode which completely simulates a single circuit and writes the output data before moving on to the next, allowing memory to be re-used. In 'batch' mode, the memory required is proportional to the number of circuits, while in 'interleaved' mode; the memory required is proportional to the size of the circuit.

Crucial to the job creation and submission system is support for data/meta-data management.

\section{4. nanoCMOS Data e-Infrastructure}

The infrastructure implemented to handle nanoCMOS simulation data consists of a repository for files and a collection of services used for managing the associated simulation metadata. The nanoCMOS data-management service is both a web service and a web interface; it is designed to be used by computational jobs to report their status and track the production of data files, and by humans (via a web browser) to query the service and monitor the progress of simulations. It provides a way to model jobs and files, and to associate them with arbitrary meta-data (dependent on the application).

The nanoCMOS infrastructure makes use of the Andrew File System (AFS), and specifically the opensource OpenAFS implementation (www.openafs.org). This provides a networked, distributed file system which maintains a single name-space across all clients. A capable role-based access control system, implemented at the directory level, coupled with the optional encryption of AFS network traffic, helps to satisfy the security needs of the project. The principal advantage of AFS is that all machines used by the project (be they service hosts, HPC nodes or user workstations) can be presented with a unified view of a "nanoCMOS file store" containing all necessary data and applications; this can be accessed almost as if it were a local file system, and thereby virtually eliminates the need to stage files.

The data-management service is based on Representational State Transfer REST principles (www.xfront.com/REST-Web-Services.html). In practice this means that every item in the system, that is every job or file, has a unique name by which it may be referenced and displayed. Similarly there are temporary identifiers for sets of search results and standard identifiers for lists of all the jobs and files and for the function to specify search criteria. The identifiers used are standard URIs, in order to facilitate access using the standard Web HTTP protocol. RESTful web services have been implemented for JobRecord and FileRecord resources to model jobs and files, respectively, as well as Annotation resources to associate meta-data with these records. In the nanoCMOS data infrastructure, a JobRecord may also have child JobRecords which are jobs spawned from the parent, e.g. to split a task over two cluster queuing systems; it may also have a number of child FileRecords, which represent the files that have been produced by the parent job. The meta-data related to jobs is populated by the job submission services.

For managing data files themselves, the datamanagement service complements the use of AFS by associating meta-data to files in AFS, by modelling them as FileRecord resources. A FileRecord holds annotations about a file, as well as a reference to it given as a URI to the file in AFS (produced as shown in Figure 3). Associating meta-data with files in AFS is performed by the jobs that produce the files, e.g. Geronimo or RandomSPICE via small clients embedded in the wrappers that run the applications. Both types of record have a set of core metadata, specific to the type, e.g. file location and type for FileRecords and job status information for JobRecords, and arbitrary applicationspecific metadata annotations. The format of these annotations is dependent upon the application that produces them. This separation allows for a 2-stage implementation of the service, supporting agile development and addressing on-going requirements of the application scientists. Because the annotations 
produced by each research application are specific to its application domain, this approach also lets the scientists retain control over choosing the metadata they need and in which format it should be produced, as opposed to a more rigid system whereby the e-Science engineer would fix the structure when designing the system, leaving little space for making the science application (often an evolving research product) change.

The query interface is embedded into the system and is accessible by pointing a web-browser at its main URI or the URI of a given record. Currently the query interfaces to the data service support core metadata-oriented querying. This allows searches amongst other things over the job type (Geronimo/RandomSPICE etc); the owner of the data; the job start and end time of the simulation; and the resource (HPC facility) where the job was run. The user interface is implemented using the Google Web Toolkit (GWT http://code.google.com/webtoolkit/). The data server offers both a web-service for programmatic clients and a user interface for browsers.

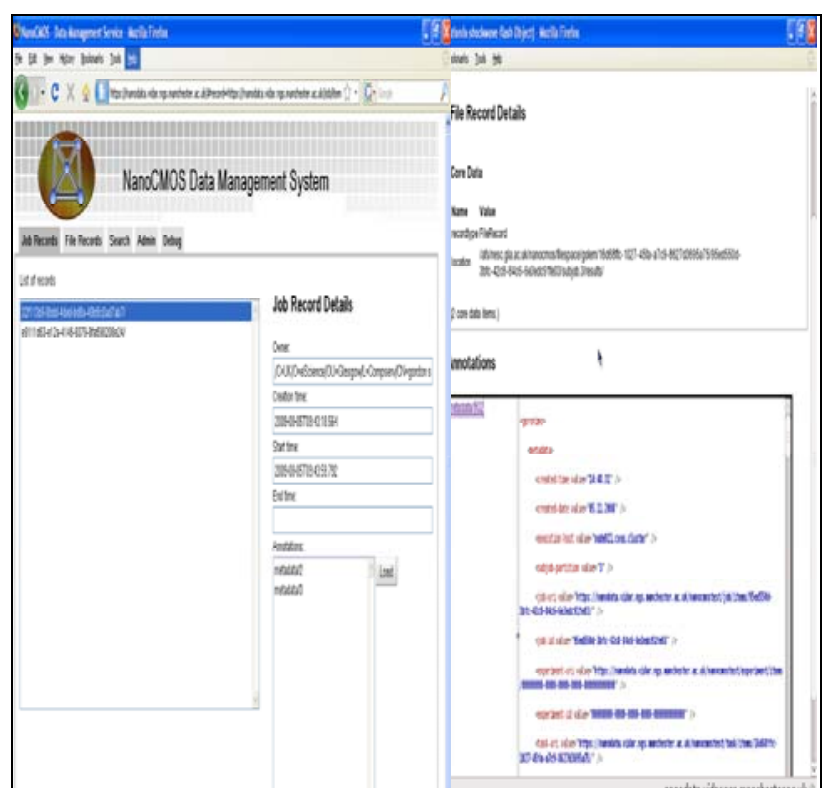

Figure 4: Data Service Interface (left) and Associated Geronimo Metadata (right)

\section{5. nanoCMOS Security e-Infrastructure}

The nanoCMOS security infrastructure has undergone numerous evolutions. Initially, the work focused upon delivery of clients via a portal. In this mode, the security was based primarily around Internet2 Shibboleth technologies (http://shibboleth.internet2.edu) to support secure federated access to and dynamic configuration of the portal contents. In this model portlets were made available depending upon the VO-specific nanoCMOS attributes that were made available as described in [20]. This exploited the results of the Open Middleware Infrastructure Institute (OMII-UK) funded SPAM-GP project (www.nesc.gla.ac.uk/projects/spam-gp). The attributes themselves could be centralised and based upon technologies such as VOMS or decentralised and stored in federated attribute authorities. These attributes could themselves be pushed or pulled to make authorisation decisions. The advantages and disadvantages of this approach for security are described in [21].

As the nanoCMOS work evolved however, and the work focused more on data security and was less portaloriented, the Shibboleth-based approach was no longer the primary focus for the e-Infrastructure security work. Instead the work was based upon seamless integration of Kerberos, GSI and VOMS-based security when accessing HPC resources, and to leverage the secure distributed file system offered by AFS.

Authentication with OpenAFS is usually performed by means of Kerberos. However, as this generally involves typing in a password-and is therefore unsuitable for use on remote resources when the user is not present-an alternative had to be sought. The chosen alternative was gssklog, a simple application which allows AFS tokens to be obtained by means of GSI. To support this, a gssklog daemon runs on one of the AFS servers, which has access to a file which maps the distinguished name (DN) of a user's e-Science X.509 certificate to a particular account within the AFS cell.

This authentication process adds an additional layer of complexity to any system which requires secure access to files stored on AFS. To perform these tasks, a series of wrapper scripts have been written (several such scripts are required because some of the internal details are resource-specific); these scripts "wrap" the actual commands to be run within additional code which ensures that AFS is available and which also completes the authentication process.

From the end users perspective, the integration of AFS, VOMS and GSI is largely transparent. When creating a job, the user is requested for a single password which is sufficient to create the Kerberos tickets and associated AFS directories, and the GSI proxy-credentials used by providers such as ScotGrid and the NGS. As noted, the mapping of nanoCMOS credentials to targeted VOspecific accounts on HPC resources through LCMAPS/LCAS is supported directly, i.e. without end users themselves necessarily being aware of this.

One further point to mention on the nanoCMOS security e-Infrastructure is with regards to data service security (and hence data security). Currently the eInfrastructure supports two primary data services: one of these is based in Glasgow and is for IP-oriented data sets specific to Glasgow device modelling, and the other is currently deployed on the UK e-Science NGS for what can loosely be described as public nanoCMOS data sets. Working is currently on-going in supporting other security-oriented data services that are hosted at partner sites. The question is whether it is feasible to host a single secure data service and exploit fine grained access control on data contents. Numerous proof of concept systems have been demonstrated that show how combinations of AFS, GSI, VOMS, PERMIS (www.permis.org) and Shibboleth can support such fine grained data access. However, it has been the case that supporting the roll-out and adoption of such solutions requires non-technical issues to be satisfactorily addressed. This is a non-trivial process. To put this in context, the project has spent approximately 2-years in getting the legal framework and agreements in place for data to be released by the commercial partners. These 
frameworks are emphatic on what can be done with the data sets and where for example the data sets might be hosted.

\section{6. nanoCMOS Case Study}

As an example case study of the utilisation of the nanoCMOS e-Infrastructure, we consider simulation of the statistical variability characterisation of a NAND gate as shown in Figure 5.

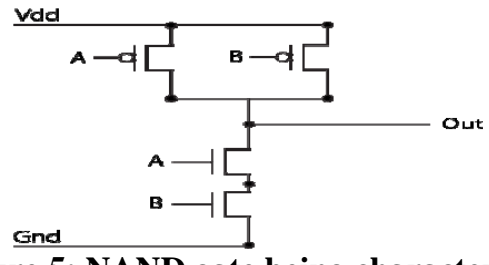

Figure 5: NAND gate being characterised

The characterisation of this NAND gate was based upon Geronimo simulations that allowed the creation of an ensemble of compact models based upon the Toshiba $35 \mathrm{~nm}$ transistor device architecture. Specifically, the simulations were based upon the quantification of the relative contributions of random discrete dopants (RDD) and line edge roughness (LER) to the device threshold voltage $\left(\mathrm{V}_{\mathrm{T}}\right)$ variability. Distributions of $\mathrm{V}_{\mathrm{T}}$ introduced by RDD and LER at low drain (VD $=100 \mathrm{mV}$ ) were compared with results as shown in Figure 8. From these simulations it is clear that while RDD is the dominant source of statistical variability in this device, LER introduces statistical variability of a comparable magnitude. Comparison of the extracted distributions with reference Gaussian distributions, having mean and standard deviation values calculated from the data, clearly shows that the LER induced distribution of $V_{T}$ is skewed in the opposite direction when compared to that of the RDD induced distribution.

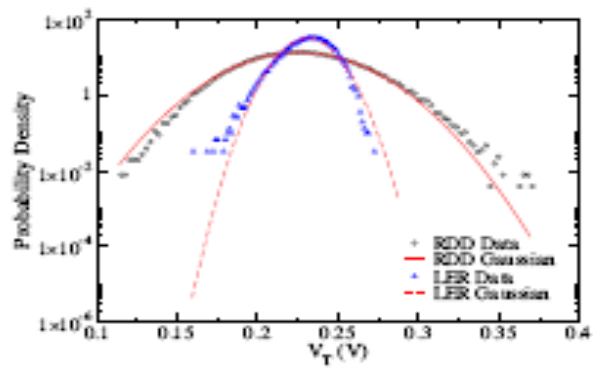

\section{Figure 6: Comparison of the histograms of $V_{T}$ obtained from RDD only and LER only simulations at $\mathrm{VD}=100 \mathrm{mV}$}

After extraction of the compact models from these devices ensembles, 50,000 simulations were carried out with RandomSPICE to characterise the cell. This simulation took approximately 1 day on 200 CPUs and generated in excess of $50 \mathrm{~Gb}$ of data. The cell itself was characterised by applying all possible combinations of inputs and input transitions. While this is only feasible for individual cells with small numbers of inputs it is necessary to obtain the correct power dissipation for the circuit, as applying single bit line transitions does not cover all the possible transitions for the cell. The energy for a transition also depends on the previous state, thus by applying all inputs we ensure that the power estimate is as accurate as possible. Delay is defined as the time from $50 \%$ on the input transition to $50 \%$ on the output transition. Obviously this is only defined for inputs the trigger an output transition.

Figure 7 shows the point cloud for the quiescent power consumption against propagation delay, showing the clouds for the slowest and fastest propagating input vectors, along with the average for all input vectors. This gives an indication of the leakage of the cell when it is inactive. It is obvious from this figure that the average energy is not actually halfway between the slowest and fastest.

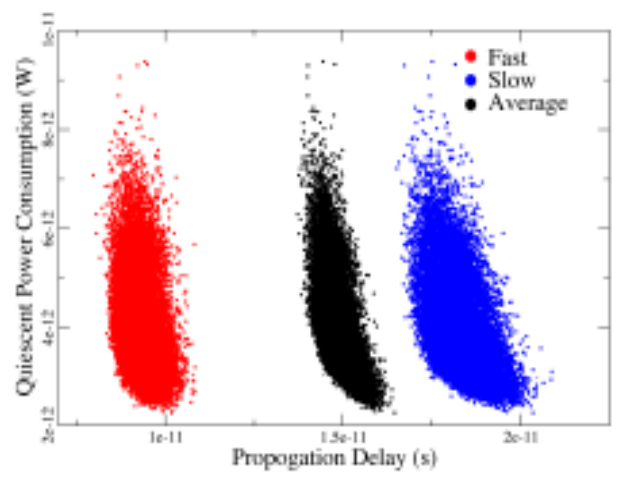

Figure 7: Quiescent power consumption of the NAND gate

\section{Conclusions}

The nanoCMOS project has developed an eInfrastructure to support research into the challenges facing the semiconductor industry caused by the atomic scale of next generation devices. A large part of the work has been based on moving from proof of concept prototypes to addressing the real scalability issues facing the electronics community. Initially it was expected that these issues would be addressed by giving seamless access to as many HPC facilities as possible. As the work evolved it was realised that data management and security represent major challenges that the eInfrastructure must address. Nevertheless the HPC demands of the nanoCMOS community have been met. The nanoCMOS VO for example, have been the primary end users of the ScotGrid HPC facility at the University of Glasgow (over 21\% total share of this resource)despite ScotGrid being predominantly configured for projects such as EGEE (Glasgow being a Tier-2 centre) and GridPP, and supporting VO's such as the ATLAS project which has over 1500 active members.

While simplistic the presented case study of a NAND gate is a perfect demonstration of proof of concept in the application of the e-Infrastructure to problems of real relevance in the electronics design community. However, the complexity of future circuits will force the evolution of these methods, in particular testing the scaling and robustness of the e-Infrastructure. Currently the integration of transistor variability into electronic system design tools that can be used to design such large-scale and complex systems is being explored. 


\subsection{Acknowledgements}

This work was funded by a grant from the UK Engineering and Physical Sciences Research Council. We gratefully acknowledge their support.

\section{References}

[1] R.O. Sinnott, et al., Meeting the Design Challenges of nanoCMOS Electronics: An Introduction to an EPSRC Pilot Project, UK e-Science All Hands Meeting, Nottingham UK, September 2006.

[2] G. Declerck, A look into the future of nanoelectronics. In VLSI Technology Technical Digest, 2005.

[3] H. P. Tuinhout. Impact of parametric mismatch and fluctuations on performance and yield of deepsubmicron CMOS technologies. In Proceedings of ESSDERC, pages 95101, 2002.

[4] H. Aikawa. Variability aware modeling and characterization in standard cell in $45 \mathrm{~nm}$ CMOS with stress enhancement technique. In VLSI Technology Symposium, pages 90-91, 2008.

[5] K. Ishimaru. 45nm/32nm CMOS challenge and perspective. In Proceedings of ESSDERC, pages 32-35, 2007.

[6] B. Cheng, S. Roy, G. Roy, F. Adamu-Lema and A. Asenov, Impact of intrinsic parameter fluctuations in decanano MOSFETs on yield and functionality of SRAM cells. Solid State Electronics, 49(5):740-746, May 2005.

[7] A. Agarwal, K. Chopra, V. Zolotov, and D. Blaauw, Circuit optimization using statistical static timing analysis. In Design Automation Conference, pages 321-324, 2005.

[8] N. Ain Kamsani, B. Cheng, et al. Statistical circuit simulation with supply voltage scaling in nanometer MOSFET devices under the influence of random dopant fluctuations. In FTFC, 2008.

[9] A. R. Brown, G. Roy, and A. Asenov, Poly-si gate-related variability in decananometer MOSFETs with conventional architecture. IEEE Transactions on Electron Devices, 54(11):3056-3063, November 2007.

[10] G. Roy, A. R. Brown, et al. Simulation study of individual and combined sources of intrinsic parameter fluctuations in conventional nano-MOSFETs. IEEE Transactions on Electron Devices, 53(12):3063-3070, December 2006.

[11] M.G. Ancona, H.F. Tiersten, Macroscopic physics of the silicon inversion layer. Phys. Rev. B, 35(15):7959-7965, 1987.

[12] S. Inaba, K. Okano, et al. High performance $35 \mathrm{~nm}$ gate length CMOS with No oxynitride gate dielectric and $\mathrm{Ni}$ salicide. IEEE Transactions on Electron Devices, 49(12):22632270, 2002.

[13] D. Reid, R.O. Sinnott, C. Millar, G. Roy, S. Roy, Gordon Stewart, Graeme Stewart, A. Asenov, Enabling Cutting-edge Semiconductor Simulation through Grid Technology, Journal of the Philosophical Transactions of the Royal Society A, July 2009, 367:2573-2584.

[14] SPICE, bwrc.eecs.berkeley.edu/Classes/icbook/SPICE/

[15] J.H. Howard, An overview of the Andrew File System, Proceedings of the USENIX 1988 Winter Technical Conference, 1988.

[16] A. Anjomshoaa et al., A. Job Submission Description Language (JSDL) specification, version 1.0., Tech. Rep. GFDR.056, Global Grid Forum, November 2005.

[17] M. Drescher et al., JSDL Parameter Sweep Extension, Tech. Rep., Open Grid Forum, September 2008.
[18] Local Centre Authorization System, http://hep-projectgrid-scg.web.cern.ch/hep-project-grid-scg/lcas-lcmaps.html

[19] R.O. Sinnott, A. Asenov, A. Brown, C. Millar, G. Roy, S. Roy, G. Stewart, Grid Infrastructures for the Electronics Domain: Requirements and Early Prototypes from an EPSRC Pilot Project, UK e-Science All Hands Meeting, Nottingham, UK, September 2007.

[20] R.O. Sinnott, D. Chadwick, T. Doherty, D. Martin, A. Stell, G. Stewart, L. Su, J. Watt, Advanced Security for Virtual Organizations: Exploring the Pros and Cons of Centralized vs Decentralized Security Models, 8th IEEE International Symposium on Cluster Computing and the Grid (CCGrid 2008), May 2008, Lyon, France.

[21] R.O. Sinnott, T. Doherty, D. Martin, C. Millar, G. Stewart, J. Watt, Supporting Security-oriented Collaborative nanoCMOS Electronics e-Research, International Conference on Computational Science, Krakow, Poland, June 2008. 


\section{University Library}

\section{- M M I N E R VA \\ A gateway to Melbourne's research publications}

Minerva Access is the Institutional Repository of The University of Melbourne

Author/s:

Sinnott, R. O.;Stewart, G.;Asenov, A.;Millar, C.;Reid, D.;Roy, G.;Roy, S.;Davenhall, C.;Harbulot, B.;Jones, M.

Title:

E-infrastructure support for nanoCMOS device and circuit simulations

Date:

2010

Citation:

Sinnott, R. O., Stewart, G., Asenov, A., Millar, C., Reid, D., Roy, G., et al. (2010). Einfrastructure support for nanoCMOS device and circuit simulations. In M. H. Hamza (Ed.), Proceedings of the Conference on Parallel and Distributed Computing and Networks, Innsbruck, Austria.

Publication Status:

Published

Persistent Link:

http://hdl.handle.net/11343/28885 